\title{
Heart Rate Variability During Hemodialysis in Patients with Chronic Renal Disease
}

овпCMRL

Sidney Benedito Silva ${ }^{1,2}$, Vitor E. Valenti ${ }^{1,4}$, Elisa Rennó Carneiro Dester ${ }^{2}$, Fernando Adami ${ }^{1}$, Yara Gracia Lorena ${ }^{2}$, David M. Garner ${ }^{3}$, Rayana L. Gomes ${ }^{4}$, Rodrigo Daminello Raimundo ${ }^{1,5}$, Ronaldo Roberto Bergamo', Tatiana Dias de Carvalho', Luiz Carlos de Abreu ${ }^{1,5}$

\section{Abstract}

Background: Few studies evaluated cardiac autonomic responses induced by hemodialysis. We aimed to evaluate cardiac autonomic regulation during hemodialysis in haemodynamically stable patients with chronic renal disease.

Method: Heart rate variability (HRV) was analyzed in the time and frequency domains in the following periods: Pre-dialysis (10 minutes before); Hemodialysis (240 minutes).

Results: The standard deviation of all normal RR intervals (SDNN) increased 60-90min after hemodialysis compared to pre-hemodialysis (29.77 $\pm 12 \mathrm{~ms}$ vs. $51.70 \pm 41.6 \mathrm{~ms}-\mathrm{p}<0.05)$. The percentage of adjacent RR intervals with a difference of duration greater than 50ms (pNN50) also raised 180-210 min after hemodialysis compared to 0-30 min after hemodialysis $(1.29 \pm 3.5$ vs. $4.37 \pm 7.8-p<0.05)$. Low frequency (LF) $\left(\mathrm{ms}^{2}\right.$ ) increased 150-180 min after hemodialysis compared to pre-hemodialysis $\left(168.2 \pm 179.8 \mathrm{~ms}^{2}\right.$ vs. $\left.562.6 \pm 455.7 \mathrm{~ms}^{2}: \mathrm{p}<0.05\right)$ and high frequency (HF) $\left(\mathrm{ms}^{2}\right)$ band raised 150-180min and 180-210 after hemodialysis compared to pre-hemodialysis $\left(59.3 \pm 70.78 \mathrm{~ms}^{2} \mathrm{vs}\right.$. $212.5 \pm 304.9 \mathrm{~ms}^{2}$ vs. $\left.186.3 \pm 335.1 \mathrm{~ms}^{2}: \mathrm{p}<0.05\right)$.

Conclusion: Hemodialysis acutely increased HRV, raising the parasympathetic component of HRV.
1 Laboratório de Delineamento de Estudos e Escrita Científica. Departamento de Saúde da Coletividade. Faculdade de Medicina do ABC, Santo André, SP, Brasil.

2 Serviço de Hemodiálise, Hospital Universitário Samuel Libânio -UNIVAS,

Pouso Alegre, MG, Brazil.

3 Cardiorespiratory Research Group. Department of Biological and Medical Sciences. Faculty of Health and Life Sciences, Oxford Brookes University.

4 Centro de Estudos do Sistema Nervoso Autônomo (CSNA), Programa de PósGraduação em Fisioterapia, Faculdade de Ciências e Tecnologia, UNESP, Presidente Prudente, SP, Brazil.

5 Departamento de Saúde Materno-Infantil. Faculdade de Saúde Pública da Universidade de São Paulo, São Paulo, Brasil.

\section{Contact information:}

\section{Sidney Benedito Silva.}

Laboratório de Delineamento de Estudos e Escrita Científica. Departamento de Saúde da Coletividade. Disciplina de Metodologia Científica. Faculdade de Medicina do ABC. Santo André, SP. Brasil.

Address: Av. Príncipe de Gales, 821, Santo André, SP CEP 09060-650.

झ" sidneybsilva@yahoo.com.br

\section{Keywords}

Autonomic nervous system; Cardiovascular system; Physiology; Renal dialysis. 


\section{Introduction}

Cardiovascular responses induced by hemodialysis, such as severe hypotension and hypertension, are considered the major clinical problematic situation during this treatment. This condition involves the autonomic dysfunction in patients with chronic renal disease [1, 2].

The investigation of cardiac autonomic regulation through heart rate variability (HRV), which evaluates the beat-to-beat spontaneous oscillations in heart rate through analysis of intervals between consecutive heartbeats (RR intervals), is a well-accepted method in the literature. HRV provides relevant clinical information regarding the state of the cardiovascular system, mainly with respect to the neural modulatory mechanism of cardiovascular function $[3,4]$.

The analysis of HRV may be performed through the time and frequency domain indices. The time domain includes the standard deviation of normalto-normal RR intervals (SDNN, indicator of global modulation), the percentage of adjacent RR intervals with a difference of duration greater than $50 \mathrm{~ms}$ (pNN50, it represents the parasympathetic component) and root-mean square of differences between adjacent normal RR intervals in a time interval (RMSSD, it corresponds to the parasympathetic modulation). The frequency domain indices include the low-frequency (LF, it is due to the joint action of the vagal and sympathetic components on the heart, with a predominance of the sympathetic component), high frequency ( $H F$, indicator of parasympathetic component) and LF/HF ratio, corresponding to the sympatho-vagal balance [4].

Previous studies reported that the normalized power of the LF component of HRV increases while the HF gradually decreases [5]. On the other hand, it was suggested that during hemodialytic hypotension, autonomic changes are different, and are characterized by parasympathetic activation and sympathetic withdrawal and, as indicated by temporaryincreases in the power of HF component [68].The main objective of our study is to describe the behavior of HRV during hemodialysis. In order to clarify this mechanism we aimed to evaluate the acute effects of hemodialysis on cardiac autonomic regulation in patients with chronic renal disease.

\section{Methods}

\section{Study Population}

We analyzed 16 haemodynamically stable patients (12 men, 4 women) with chronic renal disease under 2 to 11 years of hemodialysis treatment.The volunteers were recruited after analysis of medical treatment in the hemodialysis service of the Hospital Samuel LibanioUniversity, which included the entire medical history, associated diseases and medications.All volunteers were informed about the procedures and objectives of the study and, after agreeing, signed a term of informed consent. All procedures were approved by the Ethics Committee in Research of the Vale do Sapucai University (Protocol No. 11696912.6.0000.5102) and followed the resolution 196/96 National Health 10/10/1996.

\section{Exclusion criteria}

We considered the following exclusion criteria: neurological disorders, subjects under treatment with antibiotic and drugs that influence cardiac autonomic regulation, i.e. beta-blockers, antidepressant drugs, beta-adrenergic agonist receptors, dopaminergic agonist receptors and patients who were under hemodialysis treatment less than 3 days per week and other associated disease different from diabetes and hypertension.

\section{Initial Evaluation}

Before the experimental procedure, volunteers were identified by collecting the following information: age, gender, weight, height and body mass index (BMI). Anthropometric measurements were obtained. Body mass index (BMI) was calculated using the following formula: weight $(\mathrm{kg}) /$ height $(\mathrm{m})$ [2]. 


\section{Experimental protocol}

Data were collected in a hospital room under controlled temperature $\left(21^{\circ} \mathrm{C}-25^{\circ} \mathrm{C}\right)$ and humidity $(50 \%-60 \%)$, and volunteers were instructed to avoid consuming alcohol, caffeine and substances that influence the ANS for 24 hours before evaluation. Data were collected between 8 a.m. and 12 a.m. All procedures necessary for the data collection were explained to the individuals, and the subjects were instructed to remain at rest and not to talk during the data collection.

After the initial evaluation, the heart monitor strap was placed on each subject's thorax over the distal third of the sternum. A heart rate receiver (Polar RS800CX monitor, Polar Electro OY, Kempele, Finland), responsible for collecting the HR and RR intervals, was placed on the wrist.Systolic and diastolic arterial pressure were monitored and no significant changes were observed during hemodialysis.

\section{Data were collected in three distinct but related phases work as follows}

Pre-dialysis- at this stage HRV were recorded for 10 minutes before starting hemodialysis.

Hemodialysis- at this stage the data were collected recorded 240 minutes of hemodialysis. The hemodialysis stage was divided in 8 parts, 30 minutes each one (0-30 min, 30-60 min, 60-90 min, 90-120 min, 120-150 min, 150-180 min, 180-210 min and 210-240 min).

Post dialysis- HRV were recorded 10 minutes after hemodialysis.

\section{HRV analysis}

The RR intervals recorded by the portable HR monitor (with a sampling rate of $1000 \mathrm{~Hz}$ ) were uploaded to the Polar Precision Performance program (v. 3.0, Polar Electro, Finland). The software enabled the visualization of HR and the extraction of a cardiac period (RR interval) file in downloadable ".txt" format. Following digital filtering complemented with manual filtering for the elimination of premature ectopic beats and artifacts, at least 256 RR intervals were used for the data analysis. Only series with more than 95\% sinus rhythm was included in the study. For calculation of the indices we used the HRV Analysis software (Kubios HRV v.1.1 for Windows, Biomedical Signal Analysis Group, Department of Applied Physics, University of Kuopio, Finland) [8].

\section{Linear indices of HRV}

To analyze HRV in the frequency domain, the low frequency ( $L F=0.04$ to $0.15 \mathrm{~Hz}$ ) and high frequency ( $\mathrm{HF}=0.15$ to $0.40 \mathrm{~Hz}$ ) spectral components were used in normalized units (nu), which represents a value relative to each spectral component in relation to the total power minus the very low frequency (VLF) components, and the ratio between these components (LF/HF). The spectral analysis was calculated using the Fast Fourier Transform (FFT) algorithm.

The analysis in the time domain was performed by means of SDNN (standard deviation of normalto-normal RR intervals), the percentage of adjacent $R R$ intervals with a difference of duration greater than 50ms (pNN50) and RMSSD (root-mean square of differences between adjacent normal RR intervals in a time interval) $[8,9]$.

\section{Statistical Analysis}

Standard statistical methods were used for the calculation of means and standard deviations. Normal Gaussian distribution of the data was verified by the Shapiro-Wilk goodness-of-fit test ( $z$ value $>1.0$ ). For parametric distributions, we applied the ANOVA for repeated measures followed by the Bonferroni post-test and for non-parametric distributions we used the Friedman test followed by the Dunn's test. We compared the HRV indices between the 10 moments (pre-hemodialysis vs. 0-30 min vs. 30-60 min vs. $60-90 \mathrm{~min}$ vs. $90-120 \mathrm{~min}$ vs. $120-150 \mathrm{~min}$ vs. $150-180 \mathrm{~min}$ vs. $180-210 \mathrm{~min}$ vs. $210-240 \mathrm{~min}$ vs. post-hemodialysis). Differences were considered significant when the probability of a Type I error was less than $5 \%(p<0.05)$. We used the Software 
GraphPadStatMate version 2.00 for Windows, GraphPad Software, San Diego California USA.

\section{Results}

Data for baseline systolic (SAP) and diastolic arterial pressure (DAP), heart rate (HR) and mean RR interval, age, height, body weight and body mass index (BMI) are presented in Table 1.

We observed significant responses considering the changes of the indices in the time domain.The SDNN index showed significant increasebetween60-90 minutes after the initiation of hemodialysis compared to pre-hemodialysis.In relation to the PNN50, we noted significant increase 180-210 minutes compared to 0-30 minutes of hemodialysis (Table 2).

Regarding the LF index in absolute units, it was observed a significant increase 150-180 minutes of hemodialysis compared to pre-hemodialysis. The HF index in absolute units significantly increased 150-180minutes and 180-210 minutes of hemodialysis compared to pre-hemodialysis. On the other hand, the LF index in normalized units was not significantly changed during hemodialysis and
Table 1. Baseline diastolic (DAP) and systolic arterial pressure (SAP), heart rate (HR), mean RR interval, weight, height and body mass index (BMI) of the volunteers.

\begin{tabular}{l|c}
\multicolumn{1}{c|}{ Variable } & Value \\
\hline Age (years) & $54.4+1.7$ \\
Height $(\mathrm{m})$ & $1.7+0.1$ \\
\hline Weightbeforehemo $(\mathrm{kg})$ & $69.9+13.3$ \\
Weightafterhemo $(\mathrm{kg})$ & $64.7+13.5$ \\
\hline BMI (kg/m²) & $24.3+3.6$ \\
HR (bpm) & $78+4.4$ \\
\hline Mean RR (ms) & $793.54+39.9$ \\
SAP (mmHg) & $144+4.2$ \\
\hline DAP (mmHg) & $60+10$ \\
\hline Mean+standard-deviation. m: meters; ms: millisecond; kg: \\
kilograms; bpm: beats per minute; mmHg: millimeters of \\
mercury.
\end{tabular}

there was no significant change with respect to the $\mathrm{HF}$ in normalized units and the LF/HF ratio during hemodialysis (Table 3).

Figure 1 shows an example of the visual evaluation of the power spectrum density analysis obser-

Table 2. Absolute changes in lipid levels, liver values, and albumin values in an analysis that include data on all subjects in the relevant groups.

\begin{tabular}{|c|c|c|c|}
\hline index & SDNN & RMSSD & PNN50 \\
\hline Pre-hemodialysis & $29.77 \pm 12$ & $12.13 \pm 8$ & $1.29 \pm 3.5$ \\
\hline $0-30 \mathrm{~min}$ & $43.45 \pm 14$ & $13.23 \pm 6.8$ & $1.07 \pm 1.8$ \\
\hline 30-60min & $38.45 \pm 10$ & $14.40 \pm 5.1$ & $1.07 \pm 1.0$ \\
\hline 60-90min & $51.70 \pm 41.6 *$ & $16.73 \pm 10.1$ & $2.49 \pm 4.2$ \\
\hline 90-120min & $59.12 \pm 50.8$ & $19.97 \pm 13.7$ & $4.36 \pm 6.4$ \\
\hline 120-150min & $49.79 \pm 26$ & $20.51 \pm 15.8$ & $4.53 \pm 7.2$ \\
\hline 150-180min & $53.96 \pm 28$ & $21.71 \pm 16.4$ & $5.27 \pm 8.2$ \\
\hline 180-210min & $46.10 \pm 20$ & $18.22 \pm 15.5$ & $4.37 \pm 7.8^{* *}$ \\
\hline 210-240min & $51.46 \pm 25.9$ & $19.40 \pm 18.8$ & $3.87 \pm 7.1$ \\
\hline Post-hemodialysis & $35.73 \pm 13$ & $15.93 \pm 14.8$ & $3.57 \pm 8.7$ \\
\hline
\end{tabular}


Table 3. Mean and standard-deviation for the frequency-domain indices between, during and after hemodialysis.

\begin{tabular}{|l|c|c|c|c|c|}
\multicolumn{1}{|c|}{ Index } & LF (ms2) & LF (n.u.) & HF (ms2) & HF (nu) & LF/HF \\
\hline Pre-hemodialysis & $168.2 \pm 179.8$ & $73.8 \pm 15.1$ & $59.3 \pm 70.7$ & $26.2 \pm 15.1$ & $4.0 \pm 2.4$ \\
\hline 0-30min & $224.7 \pm 216.2$ & $77.2 \pm 11.1$ & $63.1 \pm 60.8$ & $22.8 \pm 11.15$ & $4.3 \pm 2.4$ \\
\hline 30-60min & $241.1 \pm 166.4$ & $75.3 \pm 14.2$ & $69.6 \pm 40.2$ & $24.8 \pm 14.2$ & $3.1 \pm 1.6$ \\
\hline 60-90min & $356.9 \pm 331.5$ & $76.3 \pm 10.2$ & $115.9 \pm 158.8$ & $23.7 \pm 10.19$ & $4.3 \pm 3.4$ \\
\hline 90-120min & $463.1 \pm 423.2$ & $75.5 \pm 13.6$ & $178.1 \pm 206.4$ & $24.5 \pm 13.6$ & $5.0 \pm 5.1$ \\
\hline 120-150min & $513.4 \pm 525.1$ & $76.8 \pm 11.2$ & $196.8 \pm 293.4$ & $23.2 \pm 11.2$ & $4.5 \pm 3.0$ \\
\hline 150-180min & $562.6 \pm 455.7 *$ & $77.7 \pm 11.5$ & $212.5 \pm 304.9^{*}$ & $22.3 \pm 11.5$ & $5.0 \pm 3.7$ \\
\hline 180-210min & $393.5 \pm 372.3$ & $77.9 \pm 15.9$ & $186.3 \pm 335.1 *$ & $22.1 \pm 15.9$ & $5.6 \pm 3.9$ \\
\hline 210-240min & $511.1 \pm 647.1$ & $77.9 \pm 16.6$ & $209.5 \pm 358.3$ & $22.1 \pm 16.6$ & $4.9 \pm 3.5$ \\
\hline Post-hemodialysis & $237.1 \pm 180.5$ & $74.9 \pm 17.8$ & $157.6 \pm 348.2$ & $25.1 \pm 17.8$ & $4.7 \pm 3.5$ \\
\hline LF: low frequency; HF: high frequency; LF/HF: low frequency/high frequency ratio; n.u.: normalized units; ms: \\
milliseconds. ${ }^{*}$ << 0.05: Vs 0-30 min.
\end{tabular}

ved in one subject at pre-hemodialysis (Figure 1A), 150-180 min (Figure 1B) and 180-210 min (Figure 1C) during hemodialysis and after hemodialysis (Fi- gure 1D). It is observed that the HF band is increased 50-180 min and 180-210 min during hemodialysis.

Figure 1: Example of the visual evaluation of the power spectrum density analysis observed in one subject at pre-hemodialysis (Figure 1A), 150-180 min (Figure 1B) and 180-210 min (Figure 1C) during hemodialysis and after hemodialysis (Figure 1D).
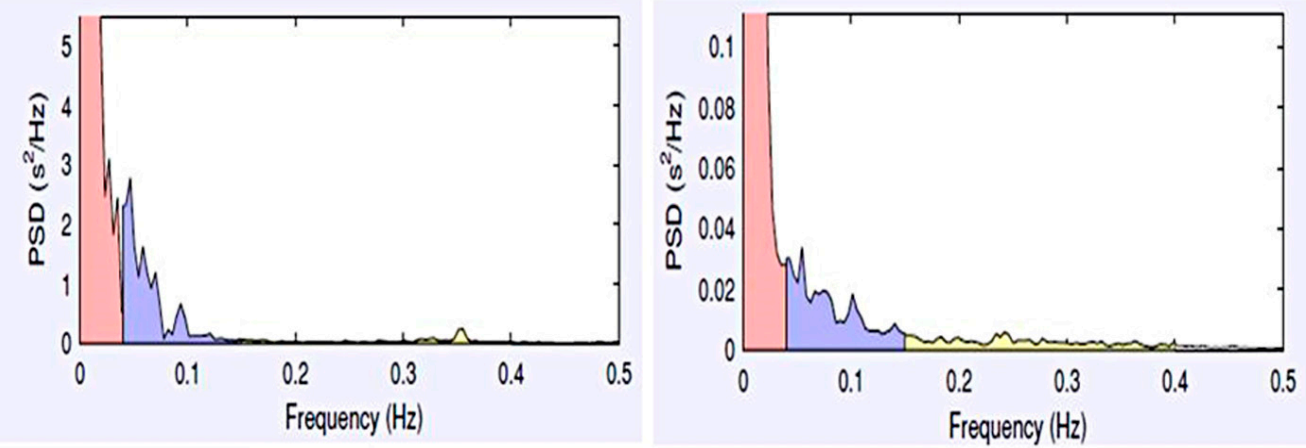

A

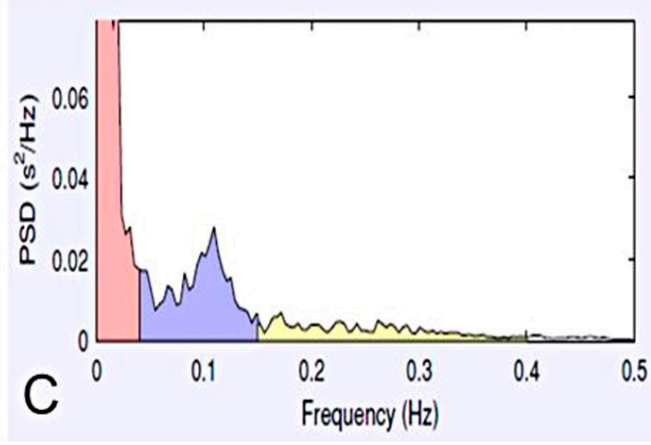

B

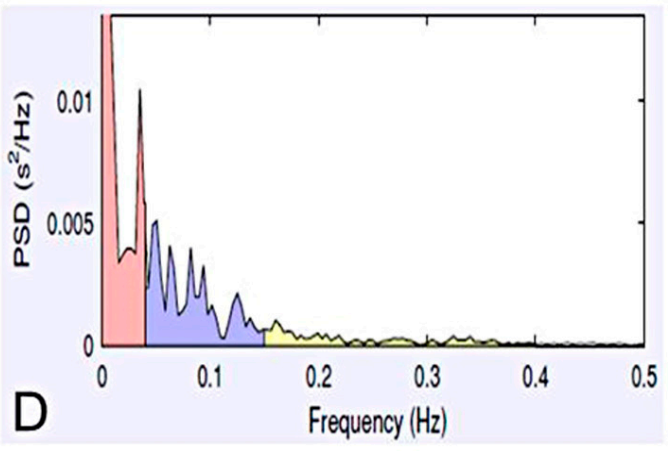




\section{Discussion}

Regulation of blood pressure and interaction of different physiological mechanisms is dependent on the autonomic nervous system. In this sense, subjects with chronic kidney disease develop dysfunctions that can lead to death. Studies using HRV presented a simple and non-invasive method with important answers in the balance of autonomic modulation in patients with this renal disorders providing sensitive information on the autonomic function and thus allowing the anticipation of larger commitments health of this population $[2,4,7]$.

Based on the clinical significant incidence of hypertension and hypotension induced by hemodialysis procedures and considering that instabilities of the renin-angiotensin-aldosterone system, plasma electrolytes levels, hematocrit as well as autonomic dysfunction were indicated to be induced by hemodialysis [1], we endeavored to investigate the cardiac autonomic regulation of haemodynamically stable subjects with chronic renal disease during hemodialysis.

We observed that HRV acutely increased around 150 and 210 minutes of hemodialysis, featured by increased time (SDNN and pNN50) and frequency (LF and HF in absolute units) domain indices. On the other hand, no significant changes were reported for systolic and diastolic arterial pressure during hemodialysis.

The SDNN index, which corresponds to the overall variability of heart rate[4], increased during hemodialysis, with a significant peak between 60-90 minutes of hemodialysis and in other periods this index also tended to increase, however, it did not reach statistical significance. During hemodialysis, adequate stimulation of sympathetic nervous system is crucial for the beginning and preservation of compensatory mechanisms to maintain blood pressure, especially peripheral vasoconstriction and an increase in heart rate $[7,10]$.The results found in our studysuggest a lower risk of a negative cardiovascular event during the hemodialysis procedure.
In this sense, according to our data, there was increase of overall variability of heart rate approximately 150-210 minutes during hemodialysis by increase in SDNN and LF in absolute units. A previous study11investigatedinter beat intervals and recorded arterial blood pressure in 93 patientswith the complex demodulation method. It was noted that no subjectpresenteddamaged consciousness or bradycardia. Moreover, they reported that hemodialysis was associated with an increased LF/HF ratio, indicatingincreased sympathetic activation.

In this context, the LF index of HRV is also considered an indicator of baroreflex function $[12,20]$. It was previously observed that during hemodialysis hypotensive events were associated with significant increases in LF and HF frequency domain indices and also in baroreflex function [11]. In the same study it was reported that the increase in baroreceptor reflex and in LF/HF ratio during hemodialysis were indicative of sympathetic nervous system activation and stimulation of the baroreflex function. Although we found no significant change in the LF/HF ratio, the findings shown in our study are in agreement with the above-mentioned results, hence, suggesting that the excessive fall of blood pressure during hemodialysis in some cases described in the literature [1] is due toinsufficient compensatory sympathetic nervous activity.

With respect to the PNN50 index we observed significant increase between 180-210minutes compared with 0-30minutes of hemodialysis. This result demonstrates that the parasympathetic modulation of the heart increased during hemodialysis. The concept of paradoxical withdrawal of sympathetic reflex vasoconstriction with bradycardias as one of the most important mechanisms of cardiovascular responsesinduced by hemodialysis $[10,12,20]$ may be suggested as a mechanism involved in the responses of the parasympathetic modulation of the heart.

Through power spectral analysis of HRV we reported that the HF in absolute units significantly increa- 
sed 150-210 minutes during hemodialysis compared to basal levels (before hemodialysis). No significant changes were noted for LF in normalized units and LF/HF ratio. In the study of Barnas et al., spectral analysis of HRV was performed during hemodialysis in patients with and without hypotension [5]. In the normotensive group, the authors reported increase in LF and decrease in HF components of HRV. This response is believed to be induced by compensatory activation of sympathetic nervous system facilitated by baroceptorreflex [13]. Conversely, in the hypotensive group it was found a decrease in LF and increase in HF components of HRV and decrease in LF/HF ratio [5]. It may be hypothesizedthat reduced sympathetic and increased parasympathetic nervous system activity and activation of the BezoldJarish, are involved in the response observed in our study. In this context, the Bezold-Jarish reflex is a sympatho-inhibitory cardio depressor reflex, which present important role in cardiovascular homeostasis $[13,19]$.Previously, von Bezold and Hirt observed that an intravenous injection of veratrum alkaloids caused a decrease in blood pressure and heart rate, which was then named the Bezold-Jarisch reflex or cardiopulmonary reflex [21].

As mentioned above, we found increase in the HF band during hemodialysis in hemodynamically stable patients with chronic renal disease. Genovesi and coworkers failed to find significant changes in this frequency domain index of HRV during hemodialysis in end-stage renal disease [15]. It was reporteda decrease in the LF and HF indices during three hours of hemodialysis compared to the prehemodialysis rest period. This study also compared hemodialysis with hemofiltration, demonstrating that the two techniquespresented variations in the variables. However, in our study we investigated the indices in each 30 minutes of hemodialysis. The importance of increase in HRV during hemodialysis, i.e. rise in the LF and HF band, as signs of improved related autonomic impairment in hemodynamically stable patients with chronic renal disease, is additio- nally corroborated by studies in patients with kidney transplantation [15].

The increased HF absolute power during hemodialysis noted in the patients evaluated in our study is an indication of a more effective oscillatory autonomic modulation of the sinoatrial node under the convective therapy regime.Some physiological mechanismsinduced by hemodialysis are suggested to be involved in the changes in HRV during our experimental procedures. There are two mechanisms involved in hemodynamic responses to hemodialysis, diffusion and ultrafiltration [16-18]. The usual response is characterized by increase in peripheral vascular resistance and cardiac output due to reduction of blood plasma volume. Initially the sympathetic nervous system is stimulated, raising heart rate, stroke volume and inducing vasoconstriction. However, this expected response was not observed in our investigation through HRV analysis. We believe that the sympathetic activation did not significantly influence the cardiac autonomic modulation in hemodynamically stable patients with renal disease and that the increase in the global modulation of the heart occurred as a response to the initial activation of the sympathetic nervous system.

This initial response of the autonomic nervous system activation is presented with cardiac protective as tonic response of the autonomic reflexes in order to stabilize cardiac functions promoting improved global functions during and after hemodialysis [18-20].

Thus, the autonomic regulation has important control of the global population homeostase during hemodyalisis as it is featured by increased survival during treatment [23], which reports that patients with kidney diseases who remain more than 2 days without treatment are more than $70 \%$ likely to die from cardiac consequences. In our study we reported the average in hemodyalisis interval, demonstrating that possibly these patients benefits continuously filtering process present favorable cardiac autonomic response. 
Our findings showed that patients with kidney disease have improved cardiac autonomic regulation during hemodialysis, supporting Rubinger et al [10] in which they reported that hemodialysis treatment induced a lower risk of sudden death or other cardiovascular aggravating event and consequently better control autonomic in chronic renal disease patients [7].

Our investigation presents some points that are worth to be raised. We did not collect plasma concentration of potassium, free calcium, epinephrine, norepinephrine and renin. Our study focused on the autonomic regulation of the heart through analysis of HRV. Future studies are suggested to evaluate the correlation of the mentioned electrolytes and plasma renin levels with the HRV indices during hemodialysis in order to evaluate the association between those variables. The patients with chronic renal disease investigated in our study presented associated diabetes and hypertension. On the other hand, patients with other associated diseases, such as neurological, cardiorespiratory and endocrine disorders that did not allow the patient to perform the experiments, were excluded.

\section{Conclusion}

Hemodialysis acutely increased global modulation of heart rate, influencing the parasympathetic component of HRV in hemodynamically stable patients with chronic renal disease with more intensity around 150-210 minutes of hemodialysis. Therefore, our data support the acute benefits of hemodialysis for cardiac autonomic regulation because it reduced the cardiac overload.

\section{Conflict of interest}

The authors have declared that no Conflict of interest exists.

\section{References}

1. Amerling $\mathrm{R}, \mathrm{Cu} \mathrm{G}$, Dubrow $\mathrm{A}$, et al. Complications during hemodialysis. In: Nissenson AR, Fine RN, Gentile DE (eds). Clinical Dialysis. 3rdedn. Appleton and Lange: Connecticut. 1995; 242-243.

2. Hildreth $C M$. Prognostic indicators of cardiovascular risk in renal disease. Frontiers in Physiology. 2010; 2: 1-6.

3. Abreu LC. Heart rate variability as a functional marker of development. J Hum Growth Developm. 2012; 22: 279-281.

4. Vanderlei LC, Pastre CM, Hoshi RA, Carvalho TD, Godoy MF. Basic notions of heart rate variability and its clinical applicability. Rev Bras Cir Cardiovasc. 2009; 24(2): 205-17.

5. Barnas MG, Boer WH, Koomans HA. Hemodynamic patterns and spectral analysis of heart rate variability during dialysis hypotension. J Am SocNephrol. 1999; 10 (12): 2577-2584.

6. Cavalcanti S, Severi S, Chiari L, et al. Autonomic nervous function during haemodialysis assessed by spectral analysis of heart-rate variability. Clin Sci (Lond) 1997; 92: 351-359.

7. Runbinger D, Revis N, Pollak A, Luria MH, Saponznikov. Predictors of haemodynamic instability and heart rate variability during hemodialysis. Nerphrol Dial Transplant. 2004; 19: 20532060.

8. Moreno IL, Pastre CM, Ferreira C, de Abreu LC, Valenti VE, Vanderlei LC. Effects of an isotonic beverage on autonomic regulation during and after exercise. J IntSoc Sports Nutr. 2013; 10(1):2.

9. Pivatelli FC, Dos Santos MA, Fernandes GB, Gatti M, de Abreu LC, Valenti VE, et. al. Sensitivity, specificity and predictive values of linear and nonlinear indices of heart rate variability in stable angina patients. Int Arch Med. 2012; 5(1):31.

10. Rubinger D, Backenroth R, Sapoznikov D. Sympathetic nervous system function and dysfunction in chronic hemodialysis patients. Semin Dial. 2013; 26(3): 333-43.

11. Sapoznikov D, Backenroth $R$, Rubinger D. Baroreflex sensitivity and sympatho-vagal balance during intradialytic hypotensive episodes. J Hypertens. 2010; 28(2): 314-324.

12. Rahman F, Pechnik S, Gross D, Sewell L, Goldstein DS. Low frequency power of heart rate variability reflects baroreflex function, not cardiac sympathetic innervation. Clin Auton Res. 2011; 21(3): 133-41.

13. Valenti VE, de Abreu LC, Imaizumi C, Petenusso M, Ferreira C. Strain differences in baroceptor reflex in adult Wistar Kyoto rats. Clinics (Sao Paulo). 2010; 65(2): 203-208.

14. Valenti VE, Abreu LC, Sato MA, Ferreira C. ATZ (3-amino-1,2,4triazole) injected into the fourth cerebral ventricle influences the Bezold-Jarisch reflex in conscious rats. Clinics (Sao Paulo). 2010; 65(12): 1339-43.

15. Genovesi S, Bracchi O, Fabbrini P, Luisetto E, Viganò MR, Lucini $D$, et. al.Differences in heart rate variability during haemodialysis and haemofiltration. Nephrol Dial Transplant. 2007; 22(8): 2256-62. 
16. Yildiz A, Sever MS, Demirel S et al. Improvement of uremic autonomic dysfunction after renal transplantation: a heart rate variabilita` study. Neprhon. 1998; 80: 57-60

17. Low PA. Autonomic nervous system function. J ClinNeurophysiol.1993; 10: 14-27.

18. Mailloux L. Hypertension in chronic renal failure and ESRD: prevalence, pathophysiology, and outcomes. SeminNephrol. 2001; 21: 146-156.

19. Antonio AM, Cardoso MA, Amaral JAT, Abreu LC, Valenti VE. Cardiac autonomic modulation adjustments in the isometric exercise. Medicalexpress 2014; 1: 351-355.

20. Fernandes TL, Piratello AC, Farah V, Fiorino P, Moreira ED, Irigoyen $\mathrm{MC}$ et al Effect of carotid and aortic baroreceptors on cardiopulmonary reflex:the role of autonomic function. Braz J Med Biol Res, 2010, 43(7) 681-686.

21. Ranpuria $R$, Hall $M$, Chan $C T$, Unruh $M$. Heart rate variability (HRV) in kidney failure: measurement and consequences of reduced HRV. Nephrol Dial Transplant. 2008; 23: 444-449.

22. Jarisch A, Zotterman Y. Depressor reflexes from the heart. Acta Physiol Scand. 1948; 16: 31-35.

23. Foley, RN, Gilbertson, D T, Murray, T, Collins, AJ. Long Interdialytic Interval and Mortalityamong Patients Receiving Hemodialysis. N Engl J Med 2011; 365: 1099-107.

\section{Comment on this article:}

\section{f] $[$ in $8+\boldsymbol{S} P$}
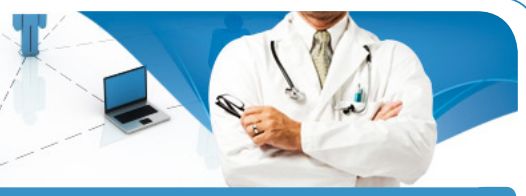

http://medicalia.org/

Where Doctors exchange clinical experiences, review their cases and share clinical knowledge. You can also access lots of medical publications for free. Join Now!

\section{Publish with iMedPub}

\section{http://www.imed.pub}

International Archives of Medicine is an open access journal publishing articles encompassing all aspects of medical science and clinical practice. IAM is considered a megajournal with independent sections on all areas of medicine. IAM is a really international journal with authors and board members from all around the world. The journal is widely indexed and classified Q1 in category Medicine. 